\title{
Outcome of bilateral circular fixators in complex lower limb fractures
}

\author{
S Mkize, ${ }^{1}$ N Ferreira ${ }^{2}$ \\ MBChB, HDip Orth (SA), FC Orth (SA), Department of Orthopaedic Surgery, Grey's Hospital, Nelson R Mandela School of Medicine, University of KwaZulu-Natal \\ BSc, MBChB, FC Orth (SA), MMed (Orth), PhD, Prof and Head Clinical Unit: Tumour, Sepsis and Reconstruction, Division of Orthopaedic Surgery, Department \\ of Surgical Sciences, Stellenbosch University, Cape Town
}

Corresponding author: Dr Sandile Mkize, Department of Orthopaedic Surgery, Grey’s Hospital, 3201, Pietermaritzburg, Email: Khabzo99@icloud.com, Tel: +27 338973000

\begin{abstract}
Circular external fixators are increasingly being used for complex lower limb trauma. When these injuries are sustained to both lower limbs, the ideal management is unclear. We present the results of 25 consecutive patients who were treated with bilateral circular external fixators for complex tibial trauma. The final cohort consisted of 23 men and two women with a mean age of 31.8 years (range 21-62 years) and a median follow-up was 16.4 months (range 6-37 months). Bony union was achieved in 96\% (48 out of 50) of the tibia fractures. The mean time to union was 26 weeks (range 13-71 weeks). Serious complications included chronic osteomyelitis that developed in one patient following a high-grade open fracture and unilateral non-unions in two patients. Bilateral circular external fixators are a viable treatment option for patients who sustain bilateral complex lower limb trauma.
\end{abstract}

Key words: bilateral circular fixators, complex tibia fractures, compound tibia fractures, soft tissue Injury, pin site sepsis

Citation: Mkize S, Ferreira N. Outcome of bilateral circular fixators in complex lower limb fractures. SAOJ 2017;16(3):51-54.

DOI 10.17159/2309-8309/2017/v16n3a7

Editor: Prof Anton Schepers, University of the Witwatersrand

Received: September 2016 Accepted: January 2017 Published: August 2017

Copyright: () Mkize S, et al. This is an open-access article distributed under the terms of the Creative Commons Attribution Licence, which permits unrestricted use, distribution and reproduction in any medium, provided the original author and source are credited.

Funding: No funding was received for the writing of this article.

Conflict of interest: Drs S Mkize and N Ferreira have no conflict of interest to declare. The content of the article is the original work of the authors. No benefits of any form have been received or will be received from a commercial party related directly or indirectly to the subject of this article.

\section{Introduction}

Complex tibial injuries are common and the ideal management is controversial. The tibia's anatomical location and subcutaneous nature makes it vulnerable during high-energy trauma, and highgrade open fractures are often the result. Non-union rates of up to $30 \%$ and infection rates of up to $40 \%$ have been reported following open fractures of the tibial diaphysis.

Apart from open fractures, peri-articular injuries to the tibia also pose significant treatment challenges to the orthopaedic surgeon tasked with their management. These injuries affect major weightbearing joints and are associated with significant morbidity. ${ }^{2,3}$ The thin soft tissue layer of the proximal and distal tibia is frequently severely injured and intolerant of extensive dissection. ${ }^{4}$

The use of circular external fixators for the management of these complex injuries has been evaluated and shown to be effective at decreasing the complications that are frequently encountered..$^{5-7}$
These fixators are attractive for the management of complex injuries due to their minimal invasive application and inherent threedimensional stability that affords early functional rehabilitation. ${ }^{8-11}$ The modularity of circular fixators further allows for frame designs specific for each patient and fracture configuration as well as the ability to address post-traumatic bone loss and limb length discrepancies.

Previous literature has been able to show the benefit of treating complex tibial injuries in circular external fixation. 5,6 When these injuries are bilateral, however, most surgeons would opt not to use bilateral circular external fixators because of the perceived morbidity and difficulty in mobilising with bilateral circular external fixators. We propose that bilateral circular external fixators would allow immediate weight bearing in patients with bilateral complex tibial fractures. The purpose of this study is to present our experience with the use of bilateral circular external fixators for bilateral complex tibia fractures. 


\section{Method}

We retrospectively reviewed all patients with acute lower limb trauma who were treated with bilateral circular external fixators between January 2007 and December 2015. Ethical approval was obtained from our institutional ethics committee before commencement of data collection. Eligible patients were identified from a prospectively collected database. Patients were excluded if they sustained major associated injuries such as spinal, pelvic and head injury. Soft tissue injures were classified according to Tscherne and Oestern for closed injuries, and Gustilo and Anderson for open injuries. ${ }^{12,13}$ Articular fractures that involved the tibial plateau were classified according to the Schatzker classification while injuries to the tibial plafond were classified according to the Ruedi and Allgower classification. ${ }^{13,14}$ Pin site infections were graded according to the Checketts and Otterburn classification. ${ }^{15}$

All patients were assessed and managed according to the Advanced Trauma Life Support (ATLS) principles on presentation. Open fractures were managed according to a standardised treatment protocol that included emergency department antibiotic administration (first generation cephalosporin and Gram-negative cover), wound irrigation and splinting. Subsequent urgent surgical debridement and temporary mono-lateral external fixation stabilisation were performed. A 48-hour wound inspection and closure was performed by either delayed primary closure, soft tissue flap or split skin graft. All patients were offered voluntary counselling and testing for human immunodeficiency virus (HIV) during their hospital admission.

Circular external fixators were individually designed according to the fracture characteristics for each patient and applied under the supervision of a consultant with limb reconstruction experience. The surgical technique followed meticulous preoperative planning and the use of pre-constructed frames. A proximal reference wire, parallel to the knee joint, followed by frame application and distal reference wire parallel to the ankle joint was placed. This aligned the fracture in the coronal plane. Sagittal plane alignment was achieved under fluoroscopy and all wires were tensioned to $130 \mathrm{Nm}$. Fixation was completed through the application of at least two tensioned wires per ring. Unstable knee injuries and tibial plafond fractures were managed by extending the frames across the knee and ankle joints respectively. Where frames were extended across joints, the extensions were removed after approximately three weeks.

Hexapod external fixators were used in cases where intentional fracture site deformity was used to facilitate soft tissue closure or where acute fracture reduction was unsuccessful. These fixators were applied using the 'rings first' method that entailed the independent, orthogonal application of the proximal and distal rings to their respective bone segments. The frame was completed by the addition of six oblique struts between the rings. Post-operative radiographs were used for planning correction of any deformity, which was commenced once the soft tissues were deemed suitable.

All patients were encouraged to mobilise full weight bearing as early as possible under the guidance of a physiotherapist. Pin site care followed a strict protocol, which involved twice-daily cleaning with a chlorhexidine in alcohol solution. ${ }^{16}$

Outpatient follow-up was scheduled at two-weekly intervals until a robust rehabilitation programme was established. Thereafter, the interval between follow-up appointments was increased to four weeks.

Radiological union was assessed by evidence of bridging callus of at least three out of four cortices. At this point, a staged 'trial of union' protocol was initiated by dynamisation of the external fixator. The site of the uniting fracture was manually stressed and if this did not cause any pain or deformity the patient was allowed to bear weight. If the patient was able to walk without pain, they were allowed to return home with a fully dynamised frame and encouraged to mobilise full weight bearing for a period of two weeks. Repeat radiographs were then compared with those before the trial of union; if no deformity had developed, union was deemed confirmed and the external fixator removed.

\section{Results}

Twenty-five patients met the inclusion criteria. No patients were excluded. The final cohort consisted of 23 men and two women with a mean age of 31.8 years (range 21-62 years). Median follow-up was 16.4 months (range 6-37 months). There were 29 (58\%) open tibia fractures (12 Gustilo-Anderson 3A and 17 Gustilo-Anderson 3B). Twelve injuries (24\%) were peri-articular and consisted of ten tibial plateau fractures and two pilon fractures. The plateau fractures consisted of seven Schatzker VI, two Schatzker IV and one Schatzker II injuries. Both pilon fractures were classified as Ruedi and Allgower type 2 injuries. All closed periarticular fractures were associated with significant soft tissue injuries. Table / illustrates the distribution of injuries.

The mechanism of injury included motor vehicle accidents in 12 patients, nine pedestrian vehicle accidents, three assaults and one fall from height. Medical comorbidities were identified in 12 patients (48\%). Ten patients (40\%) were HIV-positive with clusters of differential (CD4) counts that ranged from 153 to 1056 cells $/ \mathrm{mm}^{3}$. Eight HIV-infected patients were on Highly Active Anti-Retroviral Treatment (HAART). Twelve (48\%) patients were known smokers and one patient was diabetic and hypertensive on treatment. A single patient was a known mental health care user.

The majority of injuries (41 out of 50) were stabilised with traditional llizarov-type fine wire circular external fixators. These included 31 TrueLok fixators (Orthofix, Verona, Italy) and ten llizarov fixators (Smith \& Nephew, Memphis, Tennessee). Nine injuries were treated with hexapod circular external fixators and consisted of five Taylor Spatial Frames (Smith \& Nephew, Memphis, Tennessee) and four TrueLok-Hex fixators (Orthofix, Verona, Italy). Four tibial plateau fractures had frames that were initially spanned across the knee and two tibial pilon fractures were initially spanned across the ankle. The average time in external fixator was 26 weeks (range 13-71 weeks).

All patients attended regular follow-up and bony union was obtained in 48 out of 50 (96\%) fractures. Angular deformity was observed in two fractures post frame removal. These fractures developed less than 5 degrees valgus and varus angulation respectively and did not require further intervention. Two fractures united with slight translation at the facture site without any cosmetic or functional consequences. Two patients developed unilateral non-unions. Both patients were active smokers. These patients required a secondary procedure to achieve bony union.

The most serious complication was seen in one patient who developed chronic osteomyelitis following an open fracture. This patient was treated with chronic suppressive antibiotics and achieved union without additional surgical intervention. A single patient developed post-traumatic osteoarthritis of the knee. This patient had a complex tibial plateau (Schatzker VI) fracture which required an across-knee extension of the circular fixator. Knee stiffness post removal of the circular fixator was noted in three patients who had an initial across-knee extension of the circular fixator. All these patients required extensive physiotherapy to improve the knee range of motion.

Pin site infection developed in two of 50 (4\%) cases. Both these infections were mirror according to the Checketts, Otterburn and MacEachern classification and responded to local pin site care and oral antibiotics. ${ }^{15}$ 
Table I: Distribution of injuries

\begin{tabular}{|c|c|c|}
\hline Patient no. & Right lower limb & Left lower limb \\
\hline 1 & Proximal third tibia fracture & Proximal third compound tibia fracture (GA III A) \\
\hline 2 & Midshaft compound tibia fracture (GA III B) & Midshaft compound tibia fracture (GA III B) \\
\hline 3 & Plateau fracture (Schatzker II) & Midshaft compound tibia fracture (GA III A) \\
\hline 4 & Tibial plateau and distal femur fracture (Schatzker IV) & Distal tibia and ankle fracture \\
\hline 5 & Midshaft compound tibia fracture (GA III A) & Proximal tibia fracture with knee dislocation \\
\hline 6 & Midshaft compound tibia fracture (GA III B) & Midshaft compound tibia fracture (GA III B) \\
\hline 7 & Midshaft compound tibia fracture (GA III B) & Midshaft compound tibia fracture (GA III A) \\
\hline 8 & Midshaft compound tibia fracture (GA III A) & Distal tibia fracture closed \\
\hline 9 & Midshaft compound tibia fracture (GA III B) & Midshaft tibia fracture \\
\hline 10 & Midshaft compound tibia fracture (GA III B) & Midshaft compound tibia fracture (GA III B) \\
\hline 11 & Midshaft compound tibia fracture (GA III B) & Midshaft compound tibia fracture (GA III B) \\
\hline 12 & Midshaft compound tibia fracture (GA III A) & Midshaft compound tibia fracture (GA III A) \\
\hline 13 & Midshaft tibia fracture & Segmental tibia fracture \\
\hline 14 & Midshaft tibia fracture & Midshaft tibia fracture with knee dislocation \\
\hline 15 & Midshaft compound tibia fracture (GA III B) & Tibial plateau compound (Schatzker IV), GA III A \\
\hline 16 & Tibial plateau (Schatzker VI) and compound tibia (GA III A) & Midshaft compound tibia fracture (GA III B) \\
\hline 17 & Midshaft compound tibia fracture (GA III B) & Midshaft compound tibia fracture (GA III A) \\
\hline 18 & Tibial plateau (Schatzker VI) & Tibial plateau (Schatzker VI) \\
\hline 19 & Midshaft compound tibia fracture (GA III A) & Midshaft compound tibia fracture (GA III A) \\
\hline 20 & Segmental tibia fracture & Midshaft tibia fracture \\
\hline 21 & Tibial plafond fracture (Ruedi-Allgower II) & Tibial plafond fracture (Ruedi-Allgower II) \\
\hline 22 & Midshaft compound tibia fracture (GA III B) & Midshaft compound tibia fracture (GA III B) \\
\hline 23 & Tibial plateau (Schatzker VI) & Tibial plateau (Schatzker VI) \\
\hline 24 & Midshaft compound tibia fracture (GA III B) & Midshaft compound tibia fracture (GA III B) \\
\hline 25 & Tibial plateau (Schatzker VI) & Tibial plateau (Schatzker VI) \\
\hline
\end{tabular}

\section{Discussion}

The aim of this study was to present our experience with the use of circular external fixation for bilateral complex tibia fractures. The use of circular external fixation for deformity correction, limb lengthening, non-union management, and complex trauma is increasing exponentially. These devices are able to maintain stability and allow early mobilisation and full weight bearing. This can be achieved with minimal iatrogenic bone and soft tissue damage, preserving the remaining biological potential of the limb while simultaneously providing the mechanical environment that supports bone healing. ${ }^{17}$ Circular fixators further allow a shorter hospital stay due to earlier full weight bearing. ${ }^{18,19}$ In our case series all our patients mobilised full weight bearing with crutches immediately post-op.

Keeling et al. reviewed 67 high-energy tibial shaft fractures that were sustained secondary to war zone blast injuries. A total of 21 grade IIIA and 13 grade IIIB injuries were treated in circular fixators. All fractures healed with less than 5 degrees of coronal or sagittal malalignment at an average time of 220.8 days in external fixator. ${ }^{20}$ Ozturkmen et al. reported their results of 24 segmental tibia fractures treated in traditional llizarov-type circular fixators. Seven of these injuries were closed while the remaining 17 were open fractures (nine grade IIIA, five grade IIIB, two grade II and one grade I). The authors reported that 22 out of 24 patients achieved union with adequate alignment. Two cases united in 15-degree and 10-degree procurvatum respectively. One patient required a secondary procedure for nonunion of the middle segment of the tibia and eventually united. The mean time for proximal fracture union was 36.4 weeks while the distal fracture healed at a mean of 39.8 weeks. ${ }^{17}$ Sidharthan et al. treated 18 closed and 24 open tibia fractures (four Grade I, three Grade II, eight Grade IIIA and nine Grade IIIB) in Ilizarov-type circular external fixators. The authors reported $100 \%$ union with $92 \%$ of the cases healing with adequate alignment. All the fractures in this case series united at an average of 5.3 months (3.5 to 8.5 months). ${ }^{21}$

Our series showed bony union in 48 out of 50 fractures (96\%) at a mean time of 26 weeks (range 13-71) after the initial treatment. Two cases developed non-union that required a secondary procedure before uniting and two cases developed 5 degrees of varus and valgus post frame removal. Pin site sepsis was noted in two cases and this settled down with oral antibiotics with neither patient requiring exchange of the offending wire. One case developed chronic osteomyelitis during treatment. This patient responded to chronic suppressive antibiotics without a secondary procedure to achieve bony union.

Our results are in keeping with a number of published studies that have shown that circular fixators can be used in the treatment of complex tibia fractures. One of the primary goals of the management of open tibia fractures is to prevent infection and the infection rate in our study was 6\% (one chronic osteomyelitis and two pin site sepsis). The non-union rate in our series was 4\%; both these fractures needed a secondary procedure to achieve union. There were two cases of malunion in our series: varus and valgus deformity of five degrees. This is lower than the reported malunion rate after the use of external fixators. These patients had no functional disability, and no secondary procedures were performed to correct the deformities. In our study, no patient required removal of the circular fixator prior to bony union. 
High-energy tibial plateau fractures are serious injuries that harbour significant morbidity and demand adequate treatment and optimal rehabilitation to achieve good results. There is no universally accepted treatment for these fractures. Immediate internal fixation has some advantages although this treatment may be associated with complications such as soft tissue breakdown and chronic osteomyelitis. ${ }^{22}$ llizarov fixation of high-energy bicondylar tibial plateau fracture (Schatzker V and VI) allows early weight bearing without jeopardising fracture stability and healing. It also has the advantage, independently of fracture pattern, to operate on all patients without delay. In this way, the surgeon can avoid disturbing the healing process with other further interventions to the soft tissues, which may delay rehabilitation. ${ }^{23}$ Numerous studies have reported good to excellent results with the use of circular fixators. Kataria et al. reported their results of 38 patients who were treated with fine wire external fixators and showed no cases of non-union or septic arthritis. ${ }^{24}$ In a series by Singh et al., 20 patients with tibial plateau fracture were treated with circular fixators. The authors reported excellent results in 12 patients (60\%), good results in five patients (25\%), fair in two patients (10\%) and bad in one patient (5\%). In their series, only one case (5\%) developed non-union. ${ }^{25}$ In our study ten patients had tibial plateau fractures, three were compound fractures Gastillo-Anderson 3A and seven were closed fractures. The soft tissue injury was graded as Tscherne 2 for two fractures and Tscherne 3 for five fractures. Bony union was noted in all the fractures at an average of 20.7 weeks (14-31 weeks). Four patients had kneespanning external fixators and three patients developed knee stiffness, which responded well to extensive physiotherapy. Only one patient from our series developed post-traumatic osteoarthritis of the knee.

Pilon fractures remain an unsolved problem with various methods and philosophies of treatment. Due to the substantial risk of early soft tissue complications and risk of deep infection with open surgical repair of comminuted tibial pilon fractures, alternative methods of treatment have been investigated. ${ }^{26}$ The best results have been achieved with reconstruction of the articular surface of the tibia, stable fixation, and only a short period of joint immobilisation. ${ }^{27}$ Vidyadhara et al. reported their results of 21 consecutive patients with complex tibial pilon fractures that were treated using percutaneous reduction and fixation with llizarov circular external fixation. Nine patients from this series had open fractures. All fractures united without the need for secondary bone grafting. ${ }^{28}$ Leung et al. reviewed 31 distal tibial fractures, with 16 being classified as $C$ type $(A O)$ injuries, that were treated with a non-bridging circular external fixator. One fracture developed a non-union while another fracture was complicated by infection. ${ }^{29}$ In our case series we had two pilon fractures. Soft tissue injury in these fractures was graded as Tscherne 2 and both fractures were closed injuries. These injuries were treated with circular external fixator that crossed the ankle joint. Bony union was achieved in both fractures at 12.5 weeks (12-13 weeks) and no complications were noted.

This study has several limitations including a retrospective design, single-centre cohort and lack of a control group. Although the outcome of 50 circular external fixators is reported, the patient cohort consisted of only 25 individuals, making strong recommendations difficult.

\section{Conclusion}

The treatment of complex lower limb fracture requires a thorough knowledge of the available treatment options and their application. In rare occasions where patients sustain bilateral complex lower limb injuries, the use of bilateral circular external fixators is a viable option and can be considered without fear of negatively impacting mobilisation and rehabilitation.

\section{Compliance with Ethics Guidelines}

Ethical approval was obtained from our institutional ethics committee before commencement of data collection.

\section{References}

1. Webb LX, Bosse MJ, Castillo RC, MacKenzie EJ. Analysis of surgeon-controlled variables in the treatment of limb-threatening type-III open tibial diaphysea fractures. J Bone Joint Surg[Am]. 2007;89A:923-28.

2. Papagelopoulos PJ, Partsinevelos AA, Themistocleous GS, Mavrogenis AF, Korres DS, Soucacos PN. Complications after tibial plateau fracture surgery. Injury. 2006;37:475-84.

3. Gaston P, Will EM, Keating JF. Recovery of knee function following fracture of the tibial plateau. J Bone Joint Surg [Br]. 2005;87-B:1233-36.

4. Barei DP, Nork SE, Mills WJ, Bradford Henley M, Benirschke SK. Complications associated with internal fixation of high-energy bicondylar tibial plateau fractures utilizing a two-incision technique. J Orthop Trauma. 2004;18:649-57.

5. Dickson DR, Moulder E, Hadland Y, Giannoudis PV, Sharma HK. Grade 3 open tibial shaft fractures treated with a circular frame, functional outcome and systematic review of literature. Injury. 2015;46:751-58.

6. Ferreira N, Marais LC. Bicondylar tibial plateau fractures treated with fine wire circular external fixation. Strat Traum Limb Recon. 2014:9:25-32.

7. Ferreira N, Marais LC, Aldous C. Management of tibial non-unions: Prospective evaluation of a comprehensive treatment algorithm. SA Orthop J. 2016;15(1):60-66.

8. Podolsky A, Chao EY. Mechanical performance of llizarov circular fixators in comparison with other external fixators. Clin Orthop Relat Res. 1993;293 61-70.

9. Cunningham JL. The biomechanics of fracture fixation. Current Orthopaedics. 2001;15:457-64.

10. Caja VJ, Kim W, Larsson S, Chao EYS. Comparison of the mechanical performance of three type of external fixators: linear, circular and hybrid. Clin Biomech. 1995;10:401-406.

11. Bronson DG, Samchukov ML, Birch JG, Browne RH, Ashman RB. Stability of external circular fixation: a multi-variable biomechanical analysis. Clin Biomech. 1998;13:441-48.

12. Bode G, Strohm PC, Sudkamp NP, Hammer TO. Tibial shaft fractures management and treatment options. A review of the current literature. Acta Chir Orthop Traumatol Cech. 2012;79(6):499-505.

13. Bonar SK, Marsh JL. Tibial plafond fractures: Changing principles of treatment J Am Acad Orthop Surg. 1994;2:297-305.

14. Subramanyam NM, Rao P, Manoj-Thomas A, Mohanty K. The classification systems for tibial plateau fractures: How reliable are they? Injury. 2008;39: 1216-21.

15. Checketts RG, MacEachern AG, Otterburn M. Pin track infection and the principles of pin site care. In: De Bastiani A, Graham Apley A, Goldberg A (eds) Orthofix external fixation in trauma and orthopaedics. 2000. Springer, Berlin Heidelberg New York, pp 97-103.

16. Ferreira N, Marais LC. Pin tract sepsis: incidence with the use of circular fixators in a limb reconstruction unit. SA Orthop J. 2012;11(1):10-18.

17. Ozturkmen Y, Karamehmetoglu M, Karadeniz H, Azboy I, Caniklioglu M. Acute treatment of segmental tibial fractures with the llizarov method. Injury. 2009; 40:321-26.

18. Demiralp B, Atesalp AS, Bozkurt M, Bek D, Tasatan E, Ozturk C, Basbozkurt M. Spiral and oblique fractures of distal one-third of tibia-fibula: treatment results with circular external fixator. Ann Acad Med Singapore. 2007;36:267-71.

19. Narayan B, Marsh DR. The llizarov method in the treatment of fresh fractures. Curr Orthop. 2003;17:447-57.

20. Keeling JJ, Gwinn DE, Tintle SM, Andersen RC, McGuigan FX. Short-term outcomes of severe open wartime tibial fractures treated with ring external fixation. J Bone Joint Surg Am. 2008;90:2643-51.

21. Sidharthan S, Sujith A, Rathod A, Pathak R. Management of high energy tibial fractures using the llizarov apparatus. Internet J Orthop Surg. 2004;2(2)

22. Young MJ, Barrack RL. Complications of internal fixation of tibial plateau fractures. Orthop Rev. 1994;23:149-54.

23. Ramos T, Ekholm C, Eriksson BT, Karlsson J, Nistor L. The llizarov external fixator a useful alternative for the treatment of proximal tibial fractures. A prospective observational study of 30 consecutive patients. BMC Musculoskelet Disord. 2013;14:11

24. Kataria $\mathrm{H}$, Sharma N, Kanojia RK. Small wire external fixation for high-energy tibial plateau fractures. J Orthop Surg. 2007;15(2):137-43.

25. Singh H, Misra RK, Kaur M. Management of proximal tibia fractures using wire based circular external fixator. J Clin Diagn Res. 2015:9(9):RC01-RC04.

26. Bozkurt M, Ocguder DA, Ugurlu M, Kalkan T. Tibial pilon fracture repair using llizarov external fixation, capsuloligamentotaxis, and early rehabilitation of the ankle. J Foot Ankle Surg. 2008;47(4):302-306.

27. Koulouvaris P, Stafylas K, Mitsionis G, Vekris M, Mavrodontidis A, Xenakis T. Long-term results of various therapy concepts in severe pilon fractures. Arch Orthop Trauma Surg. 2007;127:313-20.

28. Vidyadhara S, Rao SK. llizarov treatment of complex tibial pilon fractures. Int Orthop. 2006;30:113-17.

29. Leung F, Kwok HY, Pun TS, Chow SP. Limited open reduction and llizarov external fixation in the treatment of distal tibial fractures. Injury. 2004;35 278-83. 